\title{
POBREZA INFANTIL NO BRASIL: APLICAÇÃO DA METODOLOGIA ALKIRE-FOSTER DE MENSURAÇÃO DE POBREZA MULTIDIMENSIONAL
}

\author{
Marcelo Sette Mosaner *
}

\begin{abstract}
Resumo
O presente estudo propõe exercício de medição inédita da incidência e intensidade da pobreza infantil no Brasil a partir dos dados da PNDS 2006, utilizando a metodologia elaborada por Alkire \& Foster $(2009,2011)$ para mensuração de pobreza multidimensional e sua especificação para pobreza infantil proposta por Alkire \& Roche (2012). Conclui-se que, apesar da dificuldade de obtenção de dados primários e da arbitrariedade intrínseca à escolha de dimensões em medidas multidimensionais, os benefícios da metodologia adotada superam essas adversidades em função de sua formulação robusta e intuitiva. Medidas de incidência e intensidade da pobreza são apresentadas para os estratos rural e urbano das cinco macrorregiões do Brasil, esboçando os diferentes perfis de pobreza no $\mathrm{Su}$ deste urbano e Nordeste rural.
\end{abstract}

Palavras-chave: Pobreza infantil, mensuração da pobreza multidimensional, Alkire-Foster, PNDS, DHS.

\begin{abstract}
This article proposes novel insights on the measurement of early childhood poverty incidence and intensity in Brazil, applying Alkire \& Foster $(2009,2011)$ methodology for multidimensional poverty measurement. Brazil National Demographic Women and Child Health Survey (PNDS) 2006 was used. Since PNDS is connected with worldwide Demographic Health Survey (DHS), the specification proposed by Alkire \& Roche (2012) with Bangladesh DHS (BDHS) was used to select dimensions. Despite of data and choice constraints, the benefits of Alkire Foster methodology overcome those adversities due to its robust and intuitive formulation. Measures of intensity of poverty as well as its incidence are calculated for urban and rural strata from Brazil's five macro region, drafting on rural northeast and urban southeast contrasting poverty profiles.
\end{abstract}

Keywords: Child poverty, multidimensional poverty measurement, AlkireFoster, PNDS, DHS.

JEL classification: I32

DOI: http://dx.doi.org/10.1590/1413-8050/ea 156652

* Pontifícia Universidade Católica de São Paulo. E-mail: marcelo7m@gmail.com 


\section{Siglas e Abreviações}

AC Abordagem das Capacidades, tradução de Capability Approach

AF Método Alkire-Foster

BDHS Bangladesh Demographic Health Survery

DHS Demographic Health Survery

FGT Índice Foster Greer Thorbecke

IPM/PNUD Índice de Pobreza Multidimensional / Programa das Nações Unidas para o Desenvolvimento

OMD Objetivos de Desenvolvimento do Milênio

(MDG - Millenium Development Goals)

PNDS Pesquisa Nacional da Saúde da Criança e da Mulher

USAID United States Agency for International Development 


\section{Introdução}

Este artigo propõe um exercício de mensuração da intensidade e abrangência da pobreza multidimensional em crianças de zero a cinco anos nas cinco macrorregiões do Brasil urbano e rural, com base nos dados da Pesquisa Nacional de Demografia e Saúde da Criança e da Mulher (PNDS) de 2006, realizada pelo CEBRAP e Ministério da Saúde. A metodologia utilizada foi a de nota de corte dupla proposta por Alkire \& Foster $(2009,2011)$ a partir da adaptação da aplicação feita por Alkire \& Roche (2012) para mensuração da pobreza infantil em Bangladesh, usando dados do BDHS (Bangladesh Demographic Health Survey). Tanto a PNDS brasileira como a BDHS são ligadas ao projeto Measure DHS (Demographic Health Survey), investigação global apoiada pela Agência dos Estados Unidos para o Desenvolvimento Internacional (USAID) para construção de indicadores na área de população, saúde e nutrição de mulheres e crianças em países em desenvolvimento.

Os objetivos principais deste artigo são (1) contribuir com a pesquisa e debate sobre mensuração da pobreza multidimensional, buscando uma melhor compreensão de suas vantagens e limitações; (2) introduzir o debate sobre mensuração da pobreza multidimensional infantil no Brasil e (3) avaliar a adequação do método escolhido para atingir esses objetivos ${ }^{1}$.

O artigo é dividido em cinco partes além desta introdução: (1) revisão da literatura; (2) apresentação da metodologia utilizada, condições de aplicação e resultados esperados; (3) apresentação da PNDS e adaptação de indicadores ao contexto brasileiro; (4) apresentação de resultados e (5) críticas da aplicação do modelo, restrição de dados e comentários finais.

\section{Revisão sobre mensuração da pobreza multidimensional e pobreza infantil}

Na década de setenta precipita-se uma crítica dirigida à estreiteza da medição indireta (renda) como principal métrica de desenvolvimento humano. Nesse sentido, diversos economistas elaboraram propostas de "medidas diretas" do desenvolvimento, buscando soluções alternativas à renda como proxy universal para todas as dimensões da vida humana ${ }^{2}$. Amartya Sen aponta para o reducionismo da análise econômica no contexto da economia do bem-estar (Welfare) e da Teoria da Escolha Social, apontando para dificuldades de mensuração indireta oriundas da heterogeneidade humana, como a questão das diferentes taxas de conversão de recursos e oportunidades (capabilities) em realizações (functionings). Nesse sentido, Sen esboça um arcabouço para análise de arranjos sociais baseado em ordenamentos parciais e incompletos, cujo critério primário de avaliação é a evolução do leque de oportunidades efetiva-

\footnotetext{
${ }^{1}$ Inicialmente pensou-se em realizar uma análise comparativa entre os níveis de pobreza captados pelas três edições da PNDS, realizada em 1986, 1996 e 2006. Contudo, devido às alterações significativas na metodologia entre cada edição, este artigo se limita a levantar estimativas do nível de incidência e intensidade da pobreza multidimensional em crianças de zero a cinco anos nas cinco regiões do Brasil rural e urbano, decompondo por dimensões e grupos no ano de 2006. Espera-se que seja possível tecer comparações intertemporais entre as diversas edições da PNDS numa futura contribuição.

${ }^{2}$ Dentre estes economistas podem citar os trabalhos de James Tobin, William Nordhaus, Lars Orsberg e Andrew Sharpe.
} 
mente disponíveis ao indivíduo - Capability Approach (CA). (Sen 1985, 1992, 1999).

Sen (1985) propôs uma forma de medir o desenvolvimento humano por meio de uma matriz $a_{i j}$ de $i$ vetores de capacidades e funcionamentos em $j$ vetores de dimensões para mensurar a evolução das liberdades reais dos indivíduos. Uma segunda contribuição do autor foi a concepção de que medidas de pobreza e desigualdade devem conter primeiro uma fase de identificação da população "pobre"seguida por uma segunda fase de agregação, por subgrupos ou dimensões. Ao passo que as maiores críticas à CA apontam para as dificuldades de operacionalização desse marco conceitual ${ }^{3}$, foi justamente na área da mensuração da pobreza multidimensional que emergiram as possibilidades mais relevantes de operacionalização deste marco normativo inicialmente elaborado por Sen, fruto também do desenvolvimento recente de técnicas de mensuração derivadas de medidas unidimensionais (Atkinson 2003, Bourguignon \& Chakravarty 2003, Tsui 2001). Dentre essas abordagens destaca-se a metodologia desenvolvida por Alkire \& Foster $(2011,2009)$ que propõe um conjunto de medidas intuitivas e axiomáticas de contagem de dimensões (Atkinson 2003) e derivadas do índice FGT (Foster Greer Thorbecke), que capturam não apenas a taxa de incidência $(H=$ headcount) como também a intensidade média da pobreza $(\mathrm{A}=$ average deprivation) originando a medida $M_{0}$, que é a taxa de incidência de pobreza ajustada à sua intensidade 4 . Suas principais vantagens são a capacidade de decomposição entre unidade de análise (família ou indivíduo), dimensões e regiões, seu caráter intuitivo e de fácil compreensão $\left(M_{0}\right.$ é obtido pela simples multiplicação de $H$ e $\left.A\right)$, e o respeito a axiomas centrais à medição da pobreza e desigualdade.

Diversos autores nas áreas de ciências sociais e ciências médicas têm apontado para as vantagens cognitivas e comportamentais relacionas às intervenções sociais na infância, especificamente apontado para a relação viciosa e prejudicial de privações na primeira infância ao desenvolvimento de capacidades ao longo da juventude e vida adulta ${ }^{5}$. Neste estudo, focaremos nos métodos disponíveis na literatura para identificar essas privações, realizando uma aplicação de AF a partir dos dados da PNDS.

Segundo Alkire \& Foster (2011), embora o enfoque multidimensional tenha aplicação ainda restrita na literatura sobre desenvolvimento econômico, este tem sido o enfoque predominante na literatura recente sobre pobreza infantil, estimulado por convenções e cúpulas internacionais ${ }^{6}$. A especificação proposta pelos autores utiliza a metodologia AF para mensurar não apenas a incidência da pobreza infantil $(\mathrm{H})$ - como já fora realizado no estudo da UNICEF (2007) - mas também sua intensidade (A), apresentando ao menos duas vantagens: (1) possibilita o estudo do impacto da incidência simultânea de privações em múltiplas dimensões do desenvolvimento infantil, provendo maior subsídio para políticas públicas e (2) respeita axiomas relevantes que garantem que mudanças de status nas crianças abaixo da linha de pobreza multidimensional $k$ sejam captadas.

\footnotetext{
${ }^{3}$ Flávio Comim (2008, p. 160) cita os principais críticos à capacidade de operacionalização da AC.

${ }^{4}$ Para notação matemática e prova dos axiomas consultar Alkire \& Foster (2009).

${ }^{5}$ Para mais informações, ver Shonkoff \& Phillips (2000), dentre outros.

${ }^{6}$ Alkire \& Roche (2012) citam a Convenção dos Direitos da Criança (1989), Cúpula Mundial para Crianças (1990) e a declaração "Um mundo para as Crianças" (2002).
} 
Tabela 1: Metodologia Alkire-Foster aplicada aos dados da PNDS

\begin{tabular}{|c|c|c|}
\hline Etapa & Descrição & Comentários/critério adotado \\
\hline 1 & Escolha da unidade de análise. & Crianças de até cinco anos de idade \\
\hline 2 & Escolha das dimensões de avaliação & $\begin{array}{l}\text { Acesso à água, Saneamento Básico, } \\
\text { Acesso à Habitação, Acesso à Informa- } \\
\text { ção, Saúde e Nutrição }\end{array}$ \\
\hline 3 & Escolha de variáveis para cada dimensão & $\begin{array}{l}16 \text { variáveis da PNDS, avaliando } 6 \text { di- } \\
\text { mensões (detalhadas na Tabela 2) }\end{array}$ \\
\hline 4 & $\begin{array}{l}\text { Definição de notas de cortes dimensio- } \\
\text { nais }\end{array}$ & $\begin{array}{l}\text { Definidas de acordo com literatura iden- } \\
\text { tificando os mínimos padrões aceitáveis } \\
\text { em cada dimensão (detalhes na Tabela 2) }\end{array}$ \\
\hline 5 & Atribuição de pesos relativos & $\begin{array}{l}\text { Foram atribuídos pesos iguais às seis di- } \\
\text { mensões. }\end{array}$ \\
\hline 6 & Construção da matriz censurada & $\begin{array}{l}\text { A matriz } a_{i j} \text { construída com variáveis } \\
\text { em } i \text { dimensões é censurada para pre- } \\
\text { servar a informação referente à parcela } \\
\text { identificada como "pobre"em cada di- } \\
\text { mensão, de acordo com a nota de corte } \\
\text { atribuída na etapa } 4 \text {. }\end{array}$ \\
\hline 7 & $\begin{array}{l}\text { Definição da nota de corte multidimen- } \\
\text { sional }(k)\end{array}$ & $\begin{array}{l}\text { Contagem do número de dimensões em } \\
\text { que a criança mostrou privações. Nesse } \\
\text { caso, } O \leq k \leq 6\end{array}$ \\
\hline 8 & Aplicação de $k$ & $\begin{array}{l}\text { Aplicação da nota de corte arbitrada. Re- } \\
\text { sultados são mostrados para cada valor } \\
\text { do intervalo } 1 \leq k \leq 6 \text { no Gráfico I. }\end{array}$ \\
\hline 9 & $\begin{array}{l}\text { Cálculo da incidência da pobreza ou } \\
\text { "headcount" }(H)\end{array}$ & $\begin{array}{l}\text { A incidência da pobreza } H \text { é a propor- } \\
\text { ção do número de crianças identificadas } \\
\text { como "pobres"pela nota de corte } k \text {, pelo } \\
\text { total da mostra }(n) \text {. }\end{array}$ \\
\hline 10 & $\begin{array}{l}\text { Cálculo da Intensidade média da po- } \\
\text { breza }(A)\end{array}$ & $\begin{array}{l}\text { Divisão da carga total de privações sofri- } \\
\text { das nas } 14 \text { variáveis pelo número total } \\
\text { de crianças "pobres". }\end{array}$ \\
\hline 11 & $\begin{array}{l}\text { Cálculo da incidência ajustada à intensi- } \\
\text { dade da pobreza }\left(M_{0}\right)\end{array}$ & Multiplicação entre $H(\%)$ e $A(\%)$ \\
\hline 12 & Decomposição por subgrupos & $\begin{array}{l}\text { Resultados foram decompostos regional- } \\
\text { mente em dez estratos, medindo } H, A \text {, } \\
\text { e } M_{0} \text { nas cinco macrorregiões do IBGE } \\
\text { para o Brasil urbano e rural. A decom- } \\
\text { posição foi realizada também por dimen- } \\
\text { são, comparando o Nordeste Rural com } \\
\text { o Sudeste Urbano. Por fim, foi possí- } \\
\text { vel cruzar dados sobre benefícios do go- } \\
\text { verno para a população com mais inten- } \\
\text { sidade da pobreza }(k \geq 3) \text {. }\end{array}$ \\
\hline
\end{tabular}

Fonte: Alkire \& Foster $(2009,2011)$. Elaboração do autor. 
Nesse contexto, a pobreza em crianças foi foco de estudo de Alkire \& Roche (2012), que com base em dados do BDHS (Bangladesh Demographic Health Survey) analisou a evolução na pobreza sofrida por crianças com menos de cinco anos de idade nas cinco províncias de Bangladesh entre os anos de 1997 e 2007, em quatro rodadas da pesquisa. A BDHS é apoiada pelo USAID, que também apoia a PNDS no Brasil, compartilhando parcialmente o questionário e estrutura da pesquisa. A base de dados utilizada neste estudo foi exclusivamente a PNDS 2006 (Brasil (2006)), realizada pelo CEBRAP sob os auspícios do Ministério da Saúde, que contou com informações sobre aproximadamente cinco mil crianças nos estratos urbano e rural nas cinco regiões do Brasil.

A proposta do presente estudo é mostrar as possibilidades de aplicação de AF para mensuração e análise da pobreza infantil no Brasil usando dados da PNDS 2006 - a mais recente disponível - avaliando criticamente a viabilidade dessa aplicação em função da metodologia e da base de dados disponível.

\section{Metodologia Alkire-Foster para mensuração da pobreza multidimensional}

Alkire \& Foster (2009) e Alkire \& Foster (2011) propõem uma adaptação do índice FGT à abordagem multidimensional, possibilitando mensurar incidência e intensidade da pobreza, unindo a abordagem da união e intersecção proposta por Atkinson (2003) à literatura recente de contagem aplicada à pobreza multidimensional (Tsui 2001, Bourguignon \& Chakravarty 2003, dentre outros).

A metodologia foi desenvolvida no contexto de operacionalização da Abordagem das Capacidades (AC), originalmente desenvolvida por Sen, que avalia o desenvolvimento humano em termo da evolução das capacidades (capabilities) e funcionamentos (functionings, ou realizações) efetivamente disponíveis no leque de opção de "seres" e "fazeres" de cada indivíduo. Sen propõe a construção de $i$ vetores de capacidades e $j$ dimensões compondo a matriz $a_{i j}$ de realizações (Sen 1985, 1992, 1999).

A função de identificação $\rho: R_{+}^{d} x R_{++}^{d} \rightarrow 0,1$ mapeia as realizações do indivíduo $i$ no vetor $d_{y i} \in R_{+}^{d}$ e no vetor de notas de corte $z$ em para cada variável de modo que $\rho\left(y_{i} ; z\right)=1$ se o indivíduo $i$ for pobre e $\rho\left(y_{i} ; z\right)=0$ caso contrário. A aplicação da função de identificação $\rho$ para cada vetor de realizações individuais gera o conjunto $Z \subseteq 1, \ldots, n$ de pessoas "pobres" em $y$ para dado $z$. A etapa de agregação associa $\rho$ com a matriz $y$ e o vetor de notas de corte $z$ com um nível de pobreza multidimensional $M(y ; z)$. A filtragem binária de privações 0,1 é especialmente útil para uma série de propriedades axiomáticas como o foco na pobreza e o foco em privações. A metodologia é composta de 12 passos que são detalhados em Alkire \& Foster $(2009$, 2011), Alkire \& Roche (2012) e outros e sintetizados no Quadro 1.

A primeira parte da aplicação (passos um a quatro) compreende a escolha da unidade de análise (domicílio, indivíduo ou subgrupos, nesse caso, crianças de até cinco anos), a escolha das dimensões de avaliação (no caso, as seis dimensões usadas no estudo de Alkire e Roche foram replicadas, adaptando ao caso brasileiro, conforme o Quadro 2), a escolha de variáveis em cada dimensão e as notas de cortes relativas a esses indicadores. Por exemplo, três variáveis da PNDS relativas aos índices altura-idade, altura-peso e peso-idade 
(respectivamente $\left(\right.$ XF_310INDI, XF_320INI e XF_330INDI ${ }^{7}$ foram definidas como indicador da dimensão nutricional. A nota de corte dimensional adotada é estabelecida como crianças cujo score é menor do que dois desviospadrão da série para qualquer uma das três variáveis, de acordo com a recomendação da OMS.

No quinto passo determina-se a ponderação das dimensões. Existe um longo debate acerca da metodologia para escolha de pesos em estudos multidimensionais (Decanq \& Lugo 2008), e adotamos neste estudo o critério de pesos iguais para as dimensões, seguindo o pressuposto original dos autores da metodologia, de que na falta de evidências substantivas de taxas de substituição entre dimensões e devido ao seu caráter não-hierárquico e incomensurável não há justificativa para pesos diferentes (Alkire \& Santos 2013). Uma análise mais apurada poderá prover no futuro pesos diferentes que reflitam trade-offs dentre investimentos públicos entre dimensões. O sexto passo prevê a supressão das observações de indivíduos acima da nota de corte dimensional, para tratamento apenas dos que estão abaixo da linha de pobreza ("censor matrix").Tal tratamento é essencial para cumprir com o axioma de foco na pobreza. Nos sétimo e oitavo passos a nota de corte multidimensional $k$ é definida e aplicada. Normalmente adota-se a nota média, nesse caso $k=3$. Esse parâmetro é flexível e recomenda-se na prática estudar o impacto dos vários valores de $k$ sobre a distribuição de pobreza de uma população (Gráfico 1). Os passos subsequentes tratam do cálculo de $H$ (incidência), A (intensidade) e $M_{0}$ (incidência da pobreza ajustada à intensidade). A medida " $H$ "(Headcount) é análoga à medida utilizada na abordagem unidimensional e conta a proporção de indivíduos considerados pobres em função da população total analisada. A intensidade média da pobreza (A) é obtida pela divisão da carga total de privações que cada criança está exposta individualmente pelo número total de crianças consideradas "pobres" de acordo com cada nota de corte $k$ estipulada. Segue-se (passo onze) o cálculo de $M_{0}$ pela simples multiplicação entre $H$ e $A$, provendo uma medida de incidência ajustada à intensidade da pobreza e (passo doze) exercícios de decomposição por subgrupos, de acordo com o objetivo do estudo.

Para fins ilustrativos, consta no Apêndice a decomposição de $H(n), H(\%)$, $A$ e $M_{0}$ para os 10 estratos da PNDS, compostos pelas cinco macrorregiões do Brasil segundo o IBGE, segmentados em meio urbano e rural. Por meio do cruzamento de dados com outro questionário usado pela PNDS foi possível relacionar também a quantidade de domicílios considerados "pobres" $(k \leq 3)$ que recebem benefícios do governo, como Bolsa Família, Bolsa Escola, Leite (em pó, caixinha ou saquinho), Cesta Básica, Cartão Alimentação e Auxílio Gás nos estratos urbanos, rurais e no total. Outra análise realizada foi a comparação entre quais dimensões mais afetam a pobreza multidimensional em cada estrato, fazendo comparação ilustrativa entre o caso do Nordeste Rural e o Sudeste Urbano.

A metodologia AF respeita uma série de axiomas centrais à literatura de pobreza e desigualdade, assim como suas adaptações para o enfoque multidimensional. Em síntese, esses axiomas asseguram certas propriedades relevantes à sua aplicação, como invariância à replicação, o que equivale a afirmar

\footnotetext{
${ }^{7}$ Sempre que possível mantivemos o nome original das variáveis na base de dados primários da PNDS para fins facilitar verificação dos resultados e estudos futuros. Nos casos em que foi necessário construir variáveis a partir da agregação das questões originais adotou-se a nomenclatura "XX"ao final do nome da variável, como em M493JL_XX e D027XX.
} 
que a pobreza é avaliada relativamente ao tamanho da população, garantindo que comparações corretas podem ser feitas entre populações de diferentes tamanhos e a simetria que permite que a simples troca de posição entre duas pessoas não altere a medida de pobreza (Alkire \& Foster 2009).Outro conjunto de axiomas respeitados por AF certificam que a medida reflita apenas alterações na população "pobre" sem ruídos gerados por outras alterações na população não identificada na zona de pobreza, assim como é capaz de identificar movimentações abaixo da "linha da pobreza" (Poverty Focus) captando movimentações de saída da área de pobreza justificadas por melhorias em uma ou mais dimensões (Deprivation Focus). O caráter monotônico de uma medida de pobreza tem o sentido de assegurar o nível de pobreza decresça (ou ao menos se mantenha estável) no caso de haver uma melhora na situação de indivíduos em uma determinada dimensão.

\section{Adaptação de variáveis selecionadas da PNDS para mensuração da pobreza infantil no Brasil em 2006: uma adaptação de $\mathrm{AF}$}

A PNDS é uma pesquisa direcionada à saúde da mulher com ênfase na saúde sexual, gestação e saúde da criança. Sua utilização se deve - em parte- ao exercício de replicar à especificação de Alkire \& Roche (2012) por conta do vínculo do banco de dados com a estrutura DHS, assim buscando compatibilizar a medida com a literatura internacional sobre dimensões do desenvolvimento - e em parte - e devido à insuficiência de outras bases de dados com amplitude nacional que contenham dados desagregados por criança e domicílio nas dimensões selecionadas.

Essa opção pela PNDS reflete também o objetivo de debater os problemas de adaptação de pesquisas setoriais a exercícios de mensuração da pobreza multidimensional. Devido ao enfoque recente desse tipo de análise, são poucos os censos e pesquisas domiciliares cujos questionários possam ser utilizados para esse fim. Contudo, ao combinarmos os dados dos questionários sobre o domicílio e sobre a saúde da mulher e da criança constantes da base de dados da PNDS, ainda assim foi possível reproduzir minimamente um estudo sobre pobreza multidimensional no Brasil, produzindo indicadores sintéticos sobre intensidade e abrangência da pobreza nas seis dimensões originalmente propostas por Alkire \& Roche (2012). Os dados sobre acesso à água, saneamento básico, acesso à habitação decente e acesso à informação foram extraídos da base de dados do domicílio, ao passo que os dados sobre nutrição e saúde da criança foram extraídos da base de dados sobre os filhos. As informações foram agregadas a partir da identificação dos domicílios presente no questionário dos filhos, o que possibilitou a análise de dados de 4.816 crianças.

Diversas simplificações tiveram de ser feitas para a realização deste estudo: originalmente a dimensão saúde é avaliada pela vacinação até os dois anos de idade, sendo considera em situação de privação a criança que não tiver recebido ao menos oito vacinas relacionadas pela OMS, incluindo poliomielite e BCG ou que, mesmo tendo recebido essas vacinas, não tenham recebido tratamento para diarreia ou doenças respiratórias agudas. A base de dados do PNDS 2006 não inclui dados sobre vacinação, por isso a dimensão saúde teve de ser reduzida apenas ao tratamento da diarreia para as crianças que tiveram a doença (cerca de $20 \%$ da mostra). As crianças que não receberam nenhum 
tratamento dentre todos os perguntados foram identificadas com privações na dimensão saúde, e consequentemente em estado de pobreza nessa dimensão. Nos casos em que o respondente declarou que a criança teve diarreia, mas não respondeu acerca do tratamento, também foi considerado que a criança não recebeu nenhum tratamento. Em suma, o exercício de aplicação de AlkireFoster na PNDS envolveu a adaptação das seis dimensões originalmente escolhidas para a realidade brasileira, limitando a análise aos dados disponíveis na PNDS, conforme o Quadro 2, que relaciona as variáveis originais aplicadas na BDHS e as variáveis selecionadas da PNDS brasileira, contextualizando os motivos de suas escolhas.

\section{Resultados}

O resultado do estudo é uma base dinâmica contendo dados de 4.816 crianças com capacidade de decomposição por idade, estrato, dimensão, intensidade $(A)$ e incidência $(H)$ da pobreza, assim como a incidência ajustada à intensidade $\left(M_{0}\right)$. O formato flexível de AF permite decomposição de dados de acordo com o propósito específico do estudo, respeitando o princípio de incompletude e ordenamentos parciais defendidos por Sen. Nesta seção são apresentados alguns dados obtidos neste estudo de modo a ilustrar a metodologia. O Gráfico 1 provê uma ilustração dos percentuais de $H, A$ e $M_{0}$ quando a nota de corte multidimensional $k$ varia de um a seis dimensões.

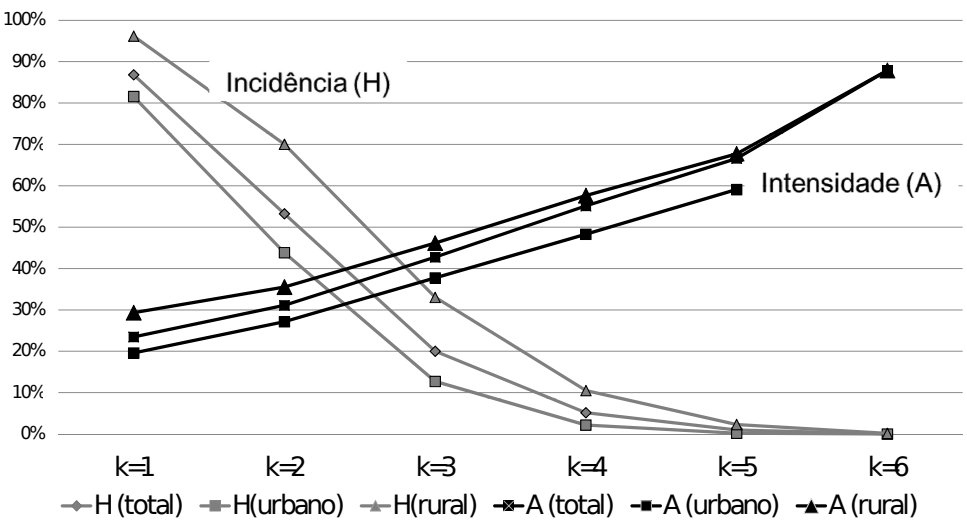

Fonte: Elaboração do autor, com dados da PNDS 2006

Figura 1: Incidência e Intensidade da pobreza para notas de corte k de uma a seis dimensões

A Tabela 3 ajuda a compreender a função de $M_{0}$ ao indicar o índice de incidência da pobreza ajustado à intensidade, ou seja, pessoas que sofrem de privações simultâneas em mais dimensões, tem maior intensidade da pobreza (A), que é a média ponderada de privações computadas individualmente em 
Tabela 2: Adaptação de critérios e notas de corte para as seis dimensões consideradas pelo estudo de Alkire e Roche (2011).

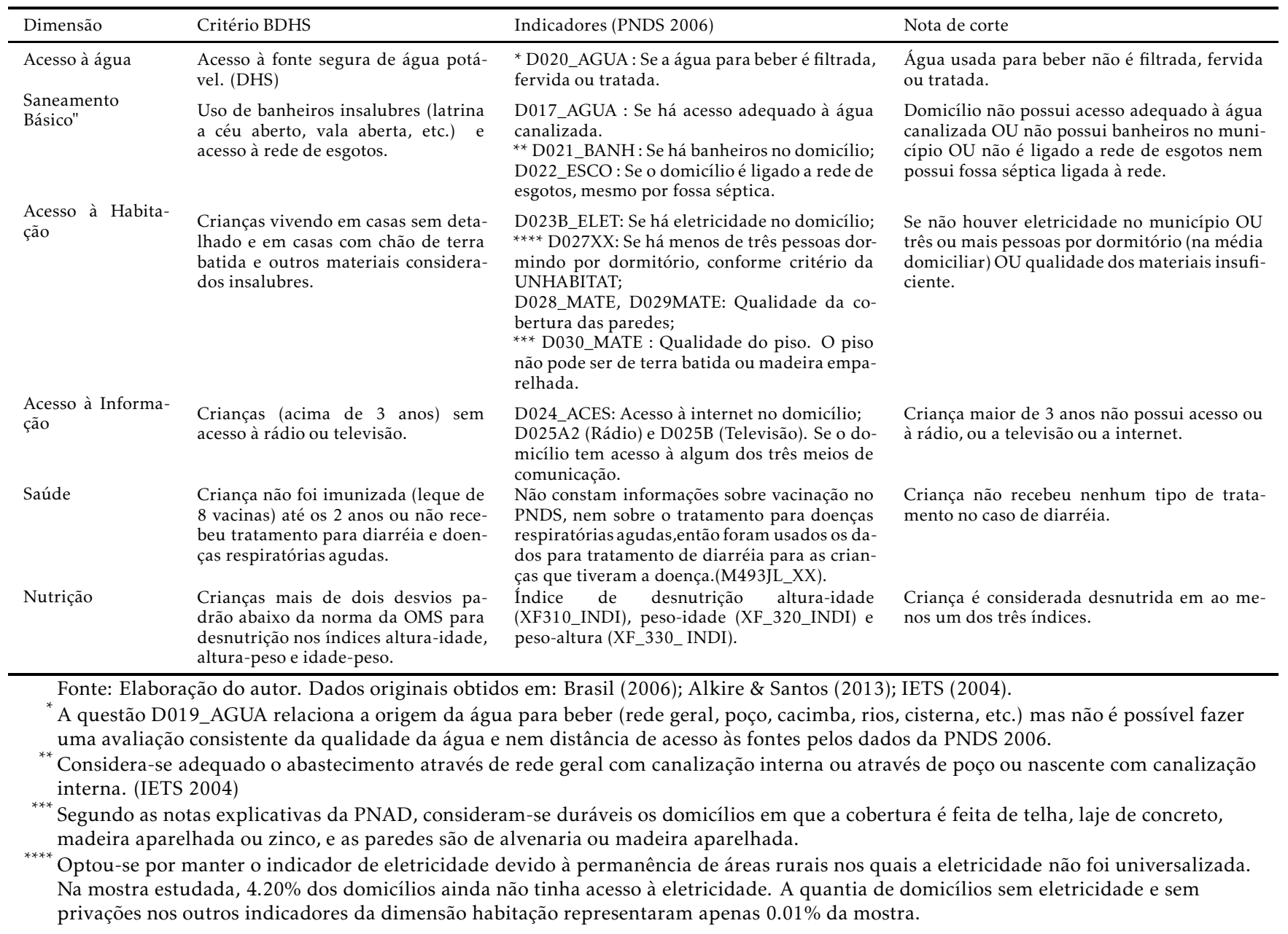

privações nos outros indicadores da dimensão habitação representaram apenas $0.01 \%$ da mostra. 
cada criança, dividida pelo número total de crianças consideradas "pobres" em múltiplas dimensões da vida. Como $M_{0}=\mathrm{HA}, M_{0}$ é sensível às alterações de status individual entre as dimensões. A segunda nota de corte $k$, que é calculada intuitivamente por meio da contagem do número de dimensões em que a criança foi identificada como pobre, pode ser usada como parâmetro de seleção de população para enfocar estudos ou políticas focadas em determinados serviços ou populações-alvo.

Tabela 3: Número de crianças identificadas como "pobres", taxa de incidência e intensidade e $M_{0}$ para seis notas de corte multidimensionais.

\begin{tabular}{lcccccc}
\hline & $k=1$ & $k=2$ & $k=3$ & $k=4$ & $k=5$ & $k=6$ \\
\hline Crianças identificadas & 4182 & 2565 & 964 & 249 & 46 & 3 \\
H (incidência) & $86,84 \%$ & $53,26 \%$ & $20,02 \%$ & $5,17 \%$ & $0,96 \%$ & $0,06 \%$ \\
A (intensidade) & $23,47 \%$ & $31,11 \%$ & $42,74 \%$ & $55,13 \%$ & $66,60 \%$ & $87,88 \%$ \\
$M_{0}$ (incidência ajus- & $20,38 \%$ & $16,5 \%$ & $8,55 \%$ & $2,85 \%$ & $0,64 \%$ & $0,05 \%$ \\
tada à intensidade) & & & & & & \\
\hline
\end{tabular}

Fonte: Elaborado pelo autor com dados da PNDS 2006.

No caso da PNDS, podemos ver que embora cerca de $87 \%$ da população sofra de privações em ao menos uma dimensão, a intensidade da pobreza nesse estrato é a menor dentre os seis estratos na Tabela 3, cerca de $23 \%$, enquanto o pequeno grupo de três crianças que sofrem de privações em todas as seis dimensões analisadas tem a maior taxa de intensidade da pobreza, com quase $88 \%$. O fato de $M_{0}$ ser calculado pela simples multiplicação de $H$ e $A$ carrega uma racionale de compensar políticas públicas que privilegiem a população mais excluída, que sofre simultaneamente um maior número de privações. Uma vez que essa medida passa a ser usada por diversos países (como o México e a Colômbia, por exemplo) é possível que haja uma focalização em torno dos mais pobres dentre os pobres, o que parece ser uma boa alternativa complementar aos programas de transferência de renda condicionados, uma vez que esses programas tendem a privilegiar a população imediatamente próxima à linha de pobreza monetária, por vezes desprezando movimentações entre dimensões e abaixo da linha de pobreza.

Nesse sentido, foi possível também traçar um perfil do grupo com maiores taxas de intensidade da pobreza que é o grupo formado por crianças que sofrem de privações em três ou mais dimensões $(k \leq 3)$. O grupo que sofre de privações em três dimensões é interessante porque, apesar de ser o terceiro grupo em termos de índice de pobreza ajustado $\left(M_{0}\right)$, com cerca de $8,55 \%$ no total, é um grupo cuja intensidade da pobreza é relativamente alta, principalmente no meio rural $(46,17 \%)$.Como resultado do estudo foi possível também identificar quais dimensões mais contribuem para a pobreza no meio urbano : quando a nota de corte $k$ é ajustada ao valor médio $k=3$, : 98,47\% das crianças encontram-se em situação de pobreza na dimensão saneamento básico, $95,15 \%$ na dimensão habitação e $56,63 \%$ na dimensão saúde, seguidos com grande distância pelas dimensões acesso à informação, com cerca de $32 \%$, nutrição, com cerca de $19,6 \%$ e acesso à água com quase $17 \%$.

O estudo aponta ainda para as relações vinculantes que dificultam a superação da pobreza (poverty trap), também conhecidas na literatura como "corrosive disavantages", indicando vínculos causais entre privações nas várias dimensões. Ainda no meio urbano, dentre as 67 crianças da mostra que são simultaneamente privadas de condições mínimas em quatro ou mais dimensões $(k \leq$ 
4), 98,5\% apresentam privações tanto na dimensão saneamento básico como em habitação, e quase três quartos delas sofrem também de pobreza privações na dimensão saúde, seguido do acesso à informação (cerca de 65\%) e nutrição (cerca de $45 \%$ ). Nenhuma das crianças identificadas na faixa de pobreza em mais de quatro privações simultâneas apresentou as notas de corte mínimas para as dimensões saneamento básico, saúde e nutrição. Já no meio rural, 182 crianças $(3,77 \%$ da mostra), sofrem privações simultaneamente em três dimensões, quase a totalidade (mais de 99\%) destas vive em situação de privação em saneamento básico e habitação, seguido de acesso à comunicação $(85 \%)$ e acesso à água para beber $(65 \%)$, caracterizando um perfil de pobreza diferente do verificado nos estratos urbanos. Após a realização de testes de sensibilidade da nota de corte $k$, é possível estabelecer outras análises uma vez que $k$ seja fixada. Para este estudo, como em muitos outros, definiu-se $k=3$, estabelecendo um parâmetro relacional médio para determinação da faixa de pobreza multidimensional ${ }^{8}$, decompondo os resultados por idade, e entre os 10 estratos da pesquisa (cinco regiões do IBGE - rural e urbano). A conclusão direta é a possibilidade de ordenamento dos 10 estratos em função da incidência, intensidade e da incidência ajustada à intensidade da pobreza $\left(M_{0}\right)$, conforme o Gráfico 2. Como pode-se constatar na maioria dos casos, a intensidade da pobreza $(A)$, medida pelo número médio de privações sofridas simultaneamente pela mesma criança, é superior à taxa de incidência em si (percentual de crianças identificadas em situação de pobreza), de modo que $M_{0}$ oferece uma perspectiva ponderada da pobreza ao combinar intensidade e incidência.

Mas em que grau essa medida se relaciona com a renda per capita familiar aferida pela mesma pesquisa? A linha da renda domiciliar per capita evidencia que embora a situação não se altere para as regiões mais prósperas - uma vez que quanto maior a renda per capita menores as medidas $H, A$ e $M_{0}$ para as regiões Sudeste, Centro-Oeste e Sul urbanos - um novo ordenamento surge entre os mais pobres e dentre os estratos intermediários, provendo novas lentes para compreensão da pobreza sob uma ótica multidimensional. Como esperado, os três estratos de maior renda per capita são os de menor intensidade da pobreza, porém, o mesmo não ocorre com os estratos mais privados da população. Embora o Norte Rural apresente renda familiar per capita média cerca de $15 \%$ superior ao Nordeste Rural ( $\mathrm{R} \$ 121,17$ contra $\mathrm{R} \$ 103,21)$, seu nível de pobreza ajustado à intensidade $M_{0}$ é de 30,76\% enquanto o do Nordeste Rural situa-se no patamar de 18,28\%. O mesmo ocorre entre outras regiões, sendo que a correlação linear entre renda familiar per capita e $M_{0}$ é de -0.741 , indicando que a renda explica apenas uma parte da pobreza multidimensional.

Outro resultado foi o cruzamento dos estratos mais pobres $(k \leq 3)$ (cerca de $20 \%$ da mostra), com os benefícios distribuídos pelo governo a esses domićllios. O Gráfico 3 mostra tal distribuição, evidenciando que no ano de realização da pesquisa o Bolsa-Família era distribuído apenas para 46,4\% dos domicílios no meio rural e para $36,4 \%$ no meio urbano, indicando possivelmente uma estratégia para direcionamento de benefícios de transferência de renda e outros auxílios.

\footnotetext{
${ }^{8} \mathrm{~A}$ escolha de $k$ é em última instância, sempre arbitrária, mas diferentes notas de corte $k$ podem ser aplicados para testes de robustez. Alkire \& Santos (2013) confirmam que as relações de dominância de primeira ordem são mantidas mesmo com diferentes valores de $k$. Nesse sentido, $k$ pode ser customizado às necessidades do estudo ou de policymakers.
} 


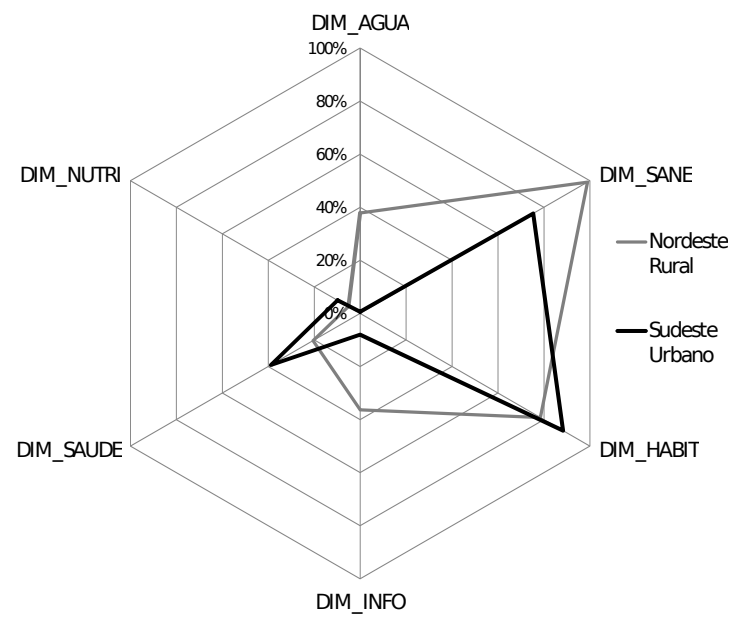

Fonte: Elaboração do autor, com dados da PNDS 2006

Figura 2: Incidência e Intensidade da pobreza e Renda domiciliar per capita (PNDS 2006)

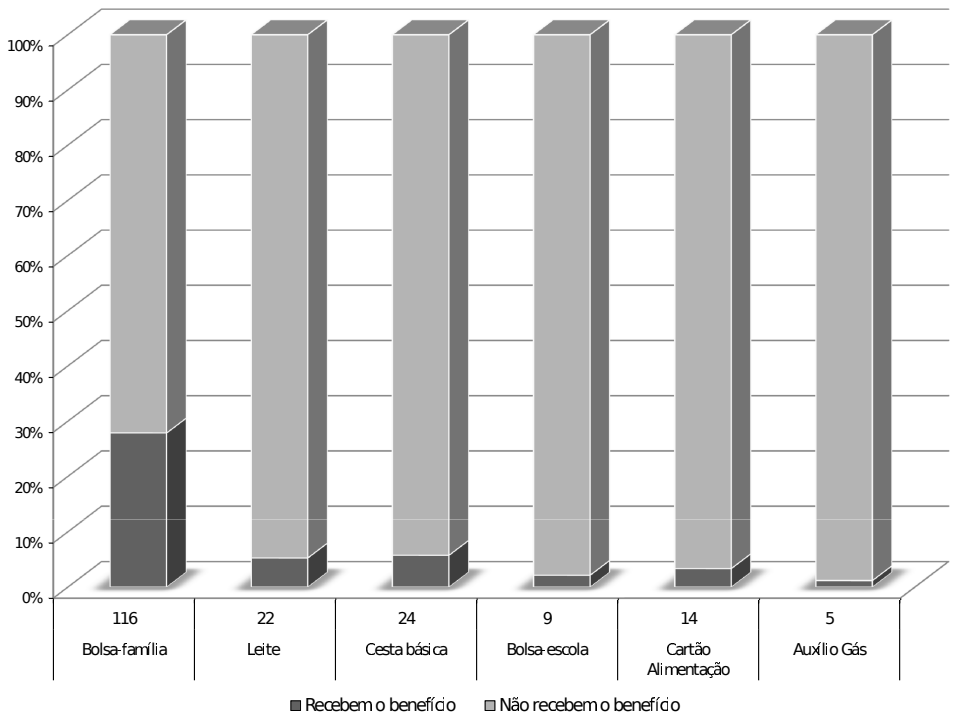

Fonte: Elaboração do autor, com dados da PNDS 2006

Figura 3: Total de domicílios identificados na faixa de pobreza multidimensional $(k \geq 3)$ que recebem benefícios do governo. 
Outro grupo de resultados é atingido se usarmos o potencial de comparação de diferentes perfis de pobreza em diferentes regiões, mostrando quais dimensões mais impactam o nível global de pobreza em determinada região. O Gráfico 4 (radar) exemplifica a propriedade de decomposição por dimensões, ilustrando que embora a área de privações do Nordeste Rural seja muito maior do que a do Sudeste Urbano, o último apresenta um maior nível de privações na dimensão Habitação, devido ao adensamento populacional, medida pelo número de pessoas por dormitório: na população avaliada, cerca de $80 \%$ das crianças do estrato SU (Sudeste Urbano) dormem em quartos com três ou mais pessoas, ao passo que esse número é de cerca de $50 \%$ para o Norte Rural. Os dados completos obtidos para $H, A$ e $M_{0}$ nos 10 estratos estudados constam no Apêndice.

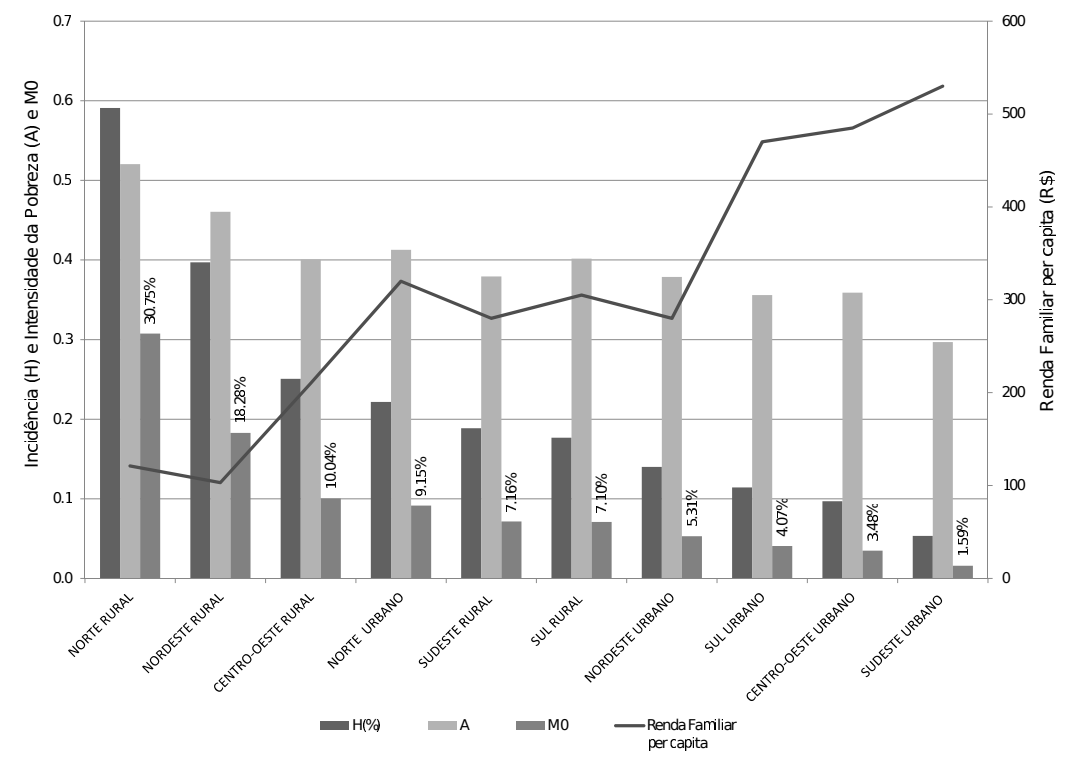

Fonte: Elaboração do autor, com dados da PNDS 2006

Figura 4: Diferentes perfis de pobreza: Nordeste Rural e Sudeste Urbano

\section{Considerações Finais}

A medida elaborada por Alkire \& Foster $(2009,2011)$ pode ser decomposta por grupo e região, apresentando a possibilidade de cálculo da intensidade pobreza entendida como a proporção de privações sofridas simultaneamente pela mesma criança.

Por meio da aplicação da metodologia foi possível aprofundar o conhecimento acerca de quais dimensões mais impactam cada estrato analisado, compreendendo as diferenças entre nível de intensidade e de incidência da pobreza multidimensional. A ferramenta é particularmente útil para fins de focalização de políticas públicas para os mais destituídos, na medida em que indica claramente quais famílias ainda não estavam sendo atendidas e em quantas dimensões cada criança sofre de privações simultâneas. Outro ponto 
forte é a possibilidade de enriquecer o debate acerca dos vínculos de causalidade da pobreza multidimensional, de modo a incentivar políticas públicas integradas no combate a privações nas várias dimensões da vida.

O presente exercício apresentou certo valor heurístico na medida em que evidenciou as dificuldades da mensuração da pobreza multidimensional em virtude da baixa disponibilidade de bases de dados com metodologia de coleta estável, informações de qualidade e periodicidade inferior a cinco anos, como ressaltado em outras aplicações de índices multidimensionais ${ }^{9}$. A dificuldade de obtenção de dados sobre o desenvolvimento na primeira infância restringe o escopo da pesquisa e seus resultados, como no caso da dimensão saúde, que neste estudo teve de ser restrita apenas ao tratamento da diarreia infantil, uma vez que a vacinação infantil não consta do questionário PNDS 2006.

O caráter arbitrário da escolha de dimensões, variáveis e pesos relativos também é um ponto de atenção na aplicação da metodologia.A robustez da nota de corte multidimensional $k$ é comumente testada, atingindo níveis satisfatórios de dominância de primeira ordem, de modo a assegurar transformações monotônicas na função de agregação ${ }^{10}$. Outros métodos são apontados para parametrizar a escolha de dimensões em índices multidimensionais, de modo a reduzir o nível de arbitrariedade (Nussbaum 2000, Alkire 2002, Nussbaum 2013). Desse modo, optamos neste estudo pela escolha de indicadores referenciados pela literatura e convenções internacionais, conforme o estudo de Alkire \& Roche (2012). Independentemente da arbitrariedade residual de medidas de pobreza, estas devem ser avaliadas pela qualidade e utilidade das informações captadas, determinando sua aplicação como ferramentas sociais de combate à pobreza, vulnerabilidade e exclusão social. Na medida em que a metodologia aplicada provê uma visão integrada do estado de privações sofridas simultaneamente na mesma criança, e enseja a criação de um mecanismo ao mesmo tempo robusto e inteligível para identificar a intensidade e a abrangência da pobreza entre subgrupos e dimensões, os benefícios apresentados pela metodologia superam em muito o caráter arbitrário das decisões metodológicas e o problema de captação de dados sociais com comparabilidade estatística. Outra contribuição natural desse tipo de exercício é justamente apontar dimensões importantes que deveriam ser incluídas na edição das próximas pesquisas em âmbito doméstico e internacional.

\section{Agradecimentos}

Agradeço pelas inúmeras contribuições do Prof. Dr. Joaquim Carlos Racy (PUC-SP) pelos valiosos comentários e apreciação crítica realizada durante o semestre acadêmico, ao Prof. Dr. Wolney Lisboa Conde do Laboratório de Avaliação Nutricional de Populações da Faculdade de Saúde Pública da USP, Eline Emanoeli (PUC-SP) pelo apoio com o software Stata 12.0 e ao Prof. Dr. Fábio Waltenberg e à Profa. Dra. Danielle Carusi Machado da Universidade

\footnotetext{
${ }^{9}$ Os autores do Índice de Pobreza Multidimensional (IPM/PNUD) chamam a atenção para a necessidade de coleta de dados válidos e normalizados para [ao menos] um número restrito de dimensões relevantes aos ODMs (Objetivos de Desenvolvimento do Milênio), dentro da mesma pesquisa estruturada em lapso de tempo inferior à cinco anos, e de preferência com a unidade de análise centrada no indivíduo, para efeito de melhor compreensão de disparidades e abuso de poder dentro do domicílio. (Alkire \& Santos 2013).

${ }^{10} \mathrm{~A}$ robustez de $k$ pode ser testada pelas estatísticas Spearman e Kendall Tau-b, como no caso do o IMP/PNUD.
} 
Federal Fluminense pelas críticas construtivas. Todos os erros e omissões são de responsabilidade exclusiva do autor.

\section{Referências Bibliográficas}

Alkire, S. (2002), 'Dimensions of human development', World Development 30(2), 180-205.

URL: http://www.sciencedirect.com/science/article/pii/S0305750X01001097

Alkire, S. \& Foster, J. (2009), Counting and multidimensional poverty. Oxford Poverty \& Human Development Initiative OPHI Working Paper n.32.

URL: http://www.ophi.org.uk/working-paper-number-32

Alkire, S. \& Foster, J. (2011), Understandings and misunderstandings of multidimensional poverty measurement. Oxford Poverty \& Human Development Initiative OPHI Working Paper n. 43.

URL: http://www.ophi.org.uk/working-paper-number-43/

Alkire, S. \& Roche, J. (2012), Beyond headcount: Measures that reflect the breadth and components of child poverty. OPHI Working Paper n. 45.

Alkire, S. \& Santos, M. (2013), Measuring acute poverty in the developing world: Robustness and scope of the multidimensional poverty index. Oxford Poverty \& Human Development Initiative Working Paper n.59.

URL: http://www.ophi.org.uk/working-paper-number-59/

Atkinson, A. (2003), 'Multidimensional deprivation: contrasting social welfare and . counting approaches', Journal of Economic Inequality 1(1), 51-65.

Bourguignon, F. \& Chakravarty, S. (2003), 'The measurement of multidimensional poverty', Journal of Economic Inequality 1(1), 25-49.

Brasil (2006), 'Pnds. base de dados da pnds 2006'.

Comim, F. (2008), Measuring capabilities, in F. Comim, M. Qizilbash \& S. Alkire, eds, 'The Capability Approach: Concepts, Measures and Applications', Cambridge University Press.

Decanq, K. \& Lugo, A. (2008), 'Setting weights in multidimensional indices of well-being', Working Paper-OPHI018 .

IETS (2004), 'Nota explicativa indicadores pnad 1992 - 2013.', Instituto de Estudos do Trabalho e Sociedade .

Nussbaum, M. (2000), Woman and Human Development: The Capabilities Approach, Cambridge University Press, Cambridge.

Nussbaum, M. (2013), Creating Capabilities: The Human Development Approach, Harvard University Press, Cambridge.

Sen, A. (1985), Commodities and Capabilities, Elsevier, Amsterdam.

Sen, A. (1992), Inequality Re-examined, Clarendon Press, Oxford.

Sen, A. (1999), Development as Freedom, AnchorBooks, New York. 
Shonkoff, J. \& Phillips, P. (2000), From Neurons to Neighborhoods:The Science of Early Childhood Development, National Academies Press, Washington, DC.

Tsui, K. (2001), 'Multidimensional poverty indices', Social Choice and Welfare 1(19), 69-93. 


\section{Apêndice A Cálculo do índice multidimensional de pobreza infantil para dez estratos da PNDS (2006)}

ESTRATO 1 - NORTE URBANO

\begin{tabular}{|c|c|c|c|c|c|c|}
\cline { 2 - 7 } \multicolumn{1}{c|}{} & $k=1$ & $k=2$ & $k=3$ & $k=4$ & $k=5$ & $k=6$ \\
\hline $\mathrm{H}(\mathrm{n})$ & 579 & 378 & 137 & 26 & 1 & 0 \\
\hline $\mathrm{N}$ & 618 & 618 & 618 & 618 & 618 & 618 \\
\hline $\mathrm{H}(\%)$ & $93,69 \%$ & $61,17 \%$ & $22,17 \%$ & $4,21 \%$ & $0,16 \%$ & - \\
\hline$\Sigma \mathrm{A}$ & 141,36 & 118,45 & 56,55 & 13,82 & 0,73 & - \\
\hline $\mathrm{A}$ & $24,42 \%$ & $31,34 \%$ & $41,27 \%$ & $53,15 \%$ & $72,73 \%$ & - \\
\hline $\mathrm{M} 0$ & $22,87 \%$ & $19,17 \%$ & $9,15 \%$ & $2,24 \%$ & $0,12 \%$ & - \\
\hline
\end{tabular}

ESTRATO 3 - NORDESTE URBANO

\begin{tabular}{|c|c|c|c|c|c|c|}
\cline { 2 - 7 } \multicolumn{1}{c|}{} & $k=1$ & $k=2$ & $k=3$ & $k=4$ & $k=5$ & $k=6$ \\
\hline $\mathrm{H}(\mathrm{n})$ & 544 & 297 & 96 & 23 & 4 & 0 \\
\hline $\mathrm{N}$ & 685 & 685 & 685 & 685 & 685 & 685 \\
\hline $\mathrm{H}(\%)$ & $79,42 \%$ & $43,36 \%$ & $14,01 \%$ & $3,36 \%$ & $0,58 \%$ & - \\
\hline$\Sigma \mathrm{A}$ & 110,00 & 83,36 & 36,36 & 10,64 & 2,18 & - \\
\hline $\mathrm{A}$ & $20,22 \%$ & $28,07 \%$ & $37,88 \%$ & $46,25 \%$ & $54,55 \%$ & - \\
\hline $\mathrm{M} 0$ & $16,06 \%$ & $12,17 \%$ & $5,31 \%$ & $1,55 \%$ & $0,32 \%$ & - \\
\hline
\end{tabular}

ESTRATO 5 - SUDESTE URBANO

\begin{tabular}{|c|c|c|c|c|c|c|}
\cline { 2 - 7 } \multicolumn{1}{c|}{} & $k=1$ & $k=2$ & $k=3$ & $k=4$ & $k=5$ & $k=6$ \\
\hline $\mathrm{H}(\mathrm{n})$ & 387 & 162 & 30 & 4 & 0 & 0 \\
\hline $\mathrm{N}$ & 561 & 561 & 561 & 561 & 561 & 561 \\
\hline $\mathrm{H}(\%)$ & $68,98 \%$ & $28,88 \%$ & $5,35 \%$ & $0,71 \%$ & - & - \\
\hline$\Sigma \mathrm{A}$ & 55,73 & 34,27 & 8,91 & 1,45 & - & - \\
\hline $\mathrm{A}$ & $14,40 \%$ & $21,16 \%$ & $29,70 \%$ & $36,36 \%$ & - & - \\
\hline $\mathrm{M} 0$ & $9,93 \%$ & $6,11 \%$ & $1,59 \%$ & $0,26 \%$ & - & - \\
\hline
\end{tabular}

ESTRATO 7 - SUL URBANO

\begin{tabular}{|c|c|c|c|c|c|c|}
\cline { 2 - 7 } \multicolumn{1}{c|}{} & $k=1$ & $k=2$ & $k=3$ & $k=4$ & $k=5$ & $k=6$ \\
\hline $\mathrm{H}(\mathrm{n})$ & 532 & 280 & 70 & 8 & 0 & 0 \\
\hline $\mathrm{N}$ & 612 & 612 & 612 & 612 & 612 & 612 \\
\hline $\mathrm{H}(\%)$ & $86,93 \%$ & $45,75 \%$ & $11,44 \%$ & $1,31 \%$ & - & - \\
\hline$\sum \mathrm{A}$ & 100,82 & 72,09 & 24,91 & 3,64 & - & - \\
\hline $\mathrm{A}$ & $18,95 \%$ & $25,75 \%$ & $35,58 \%$ & $45,45 \%$ & - & - \\
\hline $\mathrm{M} 0$ & $16,47 \%$ & $11,78 \%$ & $4,07 \%$ & $0,59 \%$ & - & - \\
\hline
\end{tabular}

ESTRATO 9 - CENTRO-OESTE URBANO

\begin{tabular}{|c|c|c|c|c|c|c|}
\cline { 2 - 7 } \multicolumn{1}{c|}{} & $k=1$ & $k=2$ & $k=3$ & $k=4$ & $k=5$ & $k=6$ \\
\hline $\mathrm{H}(\mathrm{n})$ & 474 & 235 & 59 & 6 & 1 & 0 \\
\hline $\mathrm{N}$ & 608 & 608 & 608 & 608 & 608 & 608 \\
\hline $\mathrm{H}(\%)$ & $77,96 \%$ & $38,65 \%$ & $9,70 \%$ & $0,99 \%$ & $0,16 \%$ & - \\
\hline$\Sigma \mathrm{A}$ & 84,91 & 58,82 & 21,18 & 2,82 & 0,64 & - \\
\hline $\mathrm{A}$ & $17,91 \%$ & $25,03 \%$ & $35,90 \%$ & $46,97 \%$ & $63,64 \%$ & - \\
\hline $\mathrm{M} 0$ & $13,97 \%$ & $9,67 \%$ & $3,48 \%$ & $0,46 \%$ & $0,10 \%$ & - \\
\hline
\end{tabular}

Cálculo de H, A e MO para k=3
ESTRATO 2 - NORTE RURAL

\begin{tabular}{|c|c|c|c|}
\cline { 2 - 4 } \multicolumn{1}{c|}{} & $k=1$ & $k=2$ & $k=3$ \\
\hline $\mathrm{H}(\mathrm{n})$ & 423 & 375 & 250 \\
\hline $\mathrm{N}$ & 423 & 423 & 423 \\
\hline $\mathrm{H}(\%)$ & $100,00 \%$ & $88,65 \%$ & $59,10 \%$ \\
\hline$\sum \mathrm{A}$ & 176,00 & 168,82 & 130,09 \\
\hline $\mathrm{A}$ & $41,61 \%$ & $45,02 \%$ & $52,04 \%$ \\
\hline $\mathrm{M} 0$ & $41,61 \%$ & $39,91 \%$ & $30,75 \%$ \\
\hline
\end{tabular}

ESTRATO 4 - NORDESTE RURAL

\begin{tabular}{|c|c|c|c|}
\cline { 2 - 4 } \multicolumn{1}{c|}{} & $k=1$ & $k=2$ & $k=3$ \\
\hline $\mathrm{H}(\mathrm{n})$ & 256 & 190 & 108 \\
\hline $\mathrm{N}$ & 272 & 272 & 272 \\
\hline $\mathrm{H}(\%)$ & $94,12 \%$ & $69,85 \%$ & $39,71 \%$ \\
\hline$\Sigma \mathrm{A}$ & 77,64 & 69,73 & 49,73 \\
\hline $\mathrm{A}$ & $30,33 \%$ & $36,70 \%$ & $46,04 \%$ \\
\hline $\mathrm{M} 0$ & $28,54 \%$ & $25,64 \%$ & $18,28 \%$ \\
\hline
\end{tabular}

ESTRATO 6 - SUDESTE RURAL

\begin{tabular}{|c|c|c|c|}
\cline { 2 - 4 } \multicolumn{1}{c|}{} & $k=1$ & $k=2$ & $k=3$ \\
\hline $\mathrm{H}(\mathrm{n})$ & 343 & 231 & 70 \\
\hline $\mathrm{N}$ & 371 & 371 & 371 \\
\hline $\mathrm{H}(\%)$ & $92,45 \%$ & $62,26 \%$ & $18,87 \%$ \\
\hline$\sum \mathrm{A}$ & 75,64 & 63,00 & 26,55 \\
\hline $\mathrm{A}$ & $22,05 \%$ & $27,27 \%$ & $37,92 \%$ \\
\hline $\mathrm{M} 0$ & $20,39 \%$ & $16,98 \%$ & $7,16 \%$ \\
\hline
\end{tabular}

ESTRATO 8 - SUL RURAL

\begin{tabular}{|c|c|c|c|}
\cline { 2 - 4 } \multicolumn{1}{c|}{} & $k=1$ & $k=2$ & $k=3$ \\
\hline $\mathrm{H}(\mathrm{n})$ & 307 & 203 & 55 \\
\hline $\mathrm{N}$ & 311 & 311 & 311 \\
\hline $\mathrm{H}(\%)$ & $98,71 \%$ & $65,27 \%$ & $17,68 \%$ \\
\hline$\Sigma \mathrm{A}$ & 78,91 & 63,82 & 22,09 \\
\hline $\mathrm{A}$ & $25,70 \%$ & $31,44 \%$ & $40,17 \%$ \\
\hline $\mathrm{M} 0$ & $25,37 \%$ & $20,52 \%$ & $7,10 \%$ \\
\hline
\end{tabular}

ESTRATO 10 - CENTRO-OESTE RURAL

\begin{tabular}{|c|c|c|c|}
\cline { 2 - 4 } \multicolumn{1}{c|}{} & $k=1$ & $k=2$ & $k=3$ \\
\hline $\mathrm{H}(\mathrm{n})$ & 337 & 214 & 89 \\
\hline $\mathrm{N}$ & 355 & 355 & 355 \\
\hline $\mathrm{H}(\%)$ & $94,93 \%$ & $60,28 \%$ & $25,07 \%$ \\
\hline$\Sigma \mathrm{A}$ & 80,64 & 65,64 & 35,64 \\
\hline $\mathrm{A}$ & $23,93 \%$ & $30,67 \%$ & $40,04 \%$ \\
\hline $\mathrm{M} 0$ & $22,71 \%$ & $18,49 \%$ & $10,04 \%$ \\
\hline
\end{tabular}

\begin{tabular}{|c|c|c|c|c|c|c|c|c|c|c|}
\hline & $\begin{array}{l}\text { NORTE } \\
\text { URBANO }\end{array}$ & \begin{tabular}{|l} 
NORTE \\
RURAL
\end{tabular} & $\begin{array}{l}\text { NORDESTE } \\
\text { URBANO }\end{array}$ & $\begin{array}{l}\text { NORDESTE } \\
\text { RURAL }\end{array}$ & $\begin{array}{l}\text { SUDESTE } \\
\text { URBANO }\end{array}$ & $\begin{array}{l}\text { SUDESTE } \\
\text { RURAL }\end{array}$ & $\begin{array}{c}\text { SUL } \\
\text { URBANO }\end{array}$ & $\begin{array}{c}\text { SUL } \\
\text { RURAL }\end{array}$ & $\begin{array}{l}\text { CENTRO- } \\
\text { OESTE } \\
\text { URBANO }\end{array}$ & $\begin{array}{l}\text { CENTRO- } \\
\text { OESTE } \\
\text { RURAL }\end{array}$ \\
\hline $\mathrm{N}$ & 618 & 423 & 685 & 272 & 561 & 371 & 612 & 311 & 608 & 355 \\
\hline $\mathrm{H}(\%)$ & $22,17 \%$ & $59,10 \%$ & $14,01 \%$ & $39,71 \%$ & $5,35 \%$ & $18,87 \%$ & $11,44 \%$ & $17,68 \%$ & $9,70 \%$ & $25,07 \%$ \\
\hline$\sum A$ & 56,55 & 130,09 & 36,36 & 49,73 & 8,91 & 26,55 & 24,91 & 22,09 & 21,18 & 35,64 \\
\hline$A$ & $41,27 \%$ & $52,04 \%$ & $37,88 \%$ & $46,04 \%$ & $29,70 \%$ & $37,92 \%$ & $35,58 \%$ & $40,17 \%$ & $35,90 \%$ & $40,04 \%$ \\
\hline MO & $9,15 \%$ & $30,75 \%$ & $5,31 \%$ & $18,28 \%$ & $1,59 \%$ & $7,16 \%$ & $4,07 \%$ & $7,10 \%$ & $3,48 \%$ & $10,04 \%$ \\
\hline
\end{tabular}

